\title{
Investigating the cellular origin of rotator cuff muscle fatty infiltration and fibrosis after injury
}

\author{
Xuhui Liu ${ }^{1}$ \\ Anne Y. Ning ${ }^{1}$ \\ Nai Chen Chang ${ }^{2}$ \\ Hubert Kim ${ }^{1}$ \\ Robert Nissenson ${ }^{1}$ \\ Liping Wang ${ }^{1}$ \\ Brian T. Feeley ${ }^{1}$ \\ 1 Department of Orthopaedic Surgery, University of \\ California at San Francisco, USA \\ 2 San Francisco Veterans Affairs Medical Center, \\ USA
}

Corresponding author:

Anne Y. Ning

Department of Orthopaedic Surgery, University of

California at San Francisco

1700 Owens Street Rm 361

94158 San Francisco, USA

E-mail: anneyning@gmail.com

\section{Summary}

Background: rotator cuff muscle atrophy, fibrosis and fatty infiltration are common complications after large and massive rotator cuff tears. Currently, there are no effective treatments for these muscle pathologies after injury. Furthermore, the cellular source for fibrotic and adipose tissues in rotator cuff muscle after injury remains unknown. In this study, we proposed that two groups of muscle resident progenitors, Tie2+ muscle mesenchymal progenitors and PDGFRa+ fibro/adipogenic progenitor cells (FAPs), contribute significantly to rotator cuff muscle fibrosis and fatty infiltration.

Methods: we tested our hypothesis using reporter mice. Rotator cuff muscles from Tie2-GFP and PDGFRa-GFP reporter mice were harvested at 2 and 6 weeks after unilateral massive rotator cuff tear surgeries. Immunofluorescent staining for fibroblast and adipocyte markers was conducted.

Results: our results showed significant co-localization of Tie2+ cells with fibrotic markers vimentin and aSMA. In the PDGFRa-GFP reporter mice, GFP signal was seen in only a small fraction of cells staining positive for vimentin and aSMA. However, PDGFRa showed significant co-localization with adipocyte markers, including PPAR-y, adiponectin, and perilipin $A$. Oil red $O$ staining confirmed that the mature adipocytes appearing in rotator cuff muscles after injury are also PDGFRa'.

Conclusion: these data demonstrated that the $\mathrm{Tie2}^{+}$muscle mesenchymal progenitors are the major source of fibroblasts while PDGFRa+ FAPs are the major source of adipocytes in rotator cuff muscle fatty infiltration.

Basic Science Study.

KEY WORDS: rotator cuff tear, fatty infiltration, fibrosis, fibro-adipogenic progenitors, Tie2, muscle.

\section{Introduction}

Rotator cuff $(\mathrm{RC})$ tears are one of the most common tendon injuries seen in orthopedic patients. Secondary muscle degradation, which includes muscle atrophy, fibrosis, and fatty infiltration (FI), after injury is a critical factor that determines the clinical outcome of patients. Massive RC tears have been found to be associated with atrophy and $\mathrm{FI}$ of both the supraspinatus and infraspinatus muscles in multiple studies. Poor outcomes and high re-tear rates after surgery are thought to be due in part to these changes within the muscles ${ }^{1}$, and have been demonstrated to directly correlate with the amount of muscle atrophy and $\mathrm{FI}^{2-5}$. Although fibrosis is not well described in RC injuries, recent studies have linked poor muscle function to increased expression of fibrotic markers following $\mathrm{RC}$ injury ${ }^{6,7}$. Fibrosis is also found after other muscle injuries and has been shown to directly cause muscle dysfunction in muscular dystrophy. Traditionally, it is thought that atrophy and FI are markers of end-stage $\mathrm{RC}$ disease and do not appear after smaller tears. However, recent data suggest that smaller tears can also result in atrophy and $\mathrm{FI}$ and may affect long term overall prognoses ${ }^{8}$. Patients who have disruption of the anterior supraspinatus rotator cuff cable exhibit a higher prevalence of FI in the supraspinatus than with similar tears within the central portion of the supraspinatus, even with small and medium sized tears ${ }^{8}$. Thus, muscle changes including $\mathrm{FI}$ are likely important even in smaller and medium-sized tears.

Currently, a critical gap in our understanding of the pathophysiology of fibrosis and $\mathrm{FI}$ after RC tears is knowledge of the cellular source of fibroblasts and adipocytes in RC muscle after tendon tears. Multiple stem cell and progenitor cell populations, including 
circulating bone marrow mesenchymal stem cells (BM-MSCs) and focal muscular progenitor cells, possess fibrogenic and adipogenic potential and thus could be a potential source of rotator cuff fibrosis and FI. Recent studies also proved the adipogenic potential of a Tie2+ muscle-resident progenitor cell population ${ }^{9}$. Furthermore, a novel stem cell line, termed fibro/adipogenic progenitors (FAPs), which does not arise from the myogenic lineage, was shown to be capable of giving rise to either fibroblasts or adipocytes with the correct stimulus ${ }^{10,11}$. Although these cells have a supportive role in myogenesis during development, FAPs can give rise to ectopic fibroblasts and adipocytes that accumulate in degenerating muscles. Recent studies have demonstrated that FAPs are the major source of adipocytes in muscle $\mathrm{FI}$ following other injury mechanisms ${ }^{11,12}$. Additionally, it has been demonstrated that FAPs are characterized with the surface marker platelet-derived growth factor receptor alpha (PDGFRa) in both human and rodent skeletal muscles, thus making cellular tracking of these cells possible in vivo ${ }^{13,14}$.

The goal of this study was to identify the major cellular sources of fibrosis and fatty infiltration in rotator cuff muscles after injury in a mouse model. Specifically, we focused on two primary muscle progenitor cell populations that had a high likelihood of transitioning into fibroblasts and adipocytes, investigating the contribution of $\mathrm{Tie} 2+$ progenitors and PDGFRa ${ }^{+}$ FAPs in rotator cuff muscles using GFP reporter mice. We hypothesized that either Tie2+ mesenchymal progenitors or FAP cells would be the dominant stem cell source of fibroblasts and/or adipocytes in $\mathrm{RC}$ muscles after tendon injuries in a mouse model.

\section{Materials and methods}

All research was conducted ethically according to international standards ${ }^{15}$.

\section{Reporter mouse strains}

Inducible Tie2-specific GFP-tagged transgenic mice were bred by mating mice expressing the tetracycline transactivator (tTA) under control of the endothelial lineage-selective Tie-2 promoter with tetO-HistoneGFP mice. Expression of GFP in Tie2+ progenitors was induced by maintaining the Tie2-GFP animals with tetracycline-free food following gestation.

PDGFRa-specific green fluorescent protein (GFP) transgenic reporter mice were purchased from Jackson Laboratories, Inc. (Stock \#007669, Sacramento, $\mathrm{CA})$. These mice express the H2B-eGFP fusion gene from the endogenous PDGFRa locus. Fluorescence patterns in tissues mimic the expression pattern of the endogenous gene. Previous studies have demonstrated the viability of this reporter mouse model for in vivo cell tracing for adipogenesis ${ }^{16,17}$.

\section{Massive rotator cuff tear surgery}

Twelve three-month-old PDGFRa-GFP and twelve Tie2-GFP mice (6 males and 6 females for each strain) were used in this study. In addition, 12 wildtype C57/BL6 mice were used as non-labeled controls. Massive rotator cuff tears were induced unilaterally by complete transection of the supraspinatus and infraspinatus tendons along with transection of the suprascapular nerve according to an established mouse model for massive rotator cuff tears ${ }^{18}$. Sham surgery was performed on the opposite side to serve as an internal control. All procedures were approved by our Institutional Animal Care and Use Committee.

\section{Muscular harvesting and histology}

Animals were sacrificed at two and six weeks after surgery ( 3 males and 3 females in each strain at each time point). Supraspinatus muscles from both shoulders were harvested and flash frozen in liquid nitrogen-cooled 2-methylbutane. The muscles were then cryosectioned at a thickness of $10 \mu \mathrm{m}$. Immunofluorescence (IF) staining for adipogenic markers PPAR$Y$, adiponectin, and perilipin $A$, as well as for fibrogenic markers vimentin and aSMA, was performed according to a protocol by Hemmingsen, et al. ${ }^{19}$. Sections were blocked and incubated in the respective primary antibodies overnight at a dilution range of 1:75-1:200. A rhodamine-conjugated goat-anti-rabbit secondary antibody was used at a dilution of 1:5000. After three washes in 1x PBS, sections were mounted with Vecta Shield with DAPI, a nuclear stain. Oil red $O$ staining was performed according to a protocol by Liu et al. to identify fat deposition through presence of mature adipocytes ${ }^{18}$.

\section{Image capture and quantification}

To measure the contribution of $\mathrm{GFP}^{+}$cells to adipogenesis and fibrosis, whole-section images for each muscle were captured after immunofluorescent staining with at $360 \mathrm{~nm}$ (DAPI), $480 \mathrm{~nm}$ (GFP), and 570 $\mathrm{nm}$ (rhodamine) using an Axio Observer D1 fluorescence microscope. Using image analysis software, color channels were split into blue, green, and red. $\mathrm{DAPI}^{+}, \mathrm{GFP}^{+}$, and rhodamine ${ }^{+}$cells were quantified in each muscle sample. To determine GFP/rhodamine double positive cells, images for green and red channels in each sample were overlapped. GFP ${ }^{+}$, rhodamine $^{+}$and $\mathrm{GFP}^{+} /$rhodamine $^{+}$cells were quantified manually in each section by two blinded reviewers. The percentage of GFP and rhodamine double positive cells within all rhodamine ${ }^{+}$cells in each muscle was calculated as GFP+ rhodamine $^{+}$cell number : rhodamine $^{+}$cell number $\times 100 \%$. Overlap between Tie2+ cells and adipogenic markers was negligible, and therefore not quantified. Data was run through a chi-squared test to confirm $\mathrm{p}<0.05$ and is presented as mean \pm standard error. 


\section{Results}

Using reporter mice, two muscle-resident progenitor cell populations, Tie2+ cells and PDGFRa ${ }^{+}$FAP cells, were examined for their respective abilities to differentiate into adipocytes and fibroblasts after injury (Fig. 1).

\section{Adipogenic differentiation is found only in PDGFRa cells}

Supraspinatus muscles from both Tie2-GFP reporter mice and PDGFRa-GFP reporter mice were analyzed using immunofluorescence to assess adipogenic differentiation six weeks after a massive tendon tear and denervation. Staining of Tie2-GFP sections showed that Tie2+ cells were negative for PPAR-Y and adiponectin (Fig. 2 a, b). Staining for perilipin A, a surface marker for lipid droplets, showed localization of the Tie2 signal just adjacent to the perilipin ${ }^{+}$fat cells (Fig. 2 c). Furthermore, oil red $\mathrm{O}$ stains revealed that mature adipocytes were often found adjacent to the endogenous GFP signal but did not overlap with Tie2+ cells (Fig. 3 ). This suggests that Tie2+ progenitor cells may contribute to the development of adipocytes in rotator cuff muscle $\mathrm{FI}$, but that these cell populations are not the source of fat cells.

When sections from PDGFRa-GFP reporter mice were analyzed under the same conditions, they displayed strong overlap between the endogenous GFP signal and stains for adipogenic markers (Fig. 4 a-c). Quantification of fluorescence showed that $96.10 \pm$
$3.27 \%$ of all cells expressing the late-stage adipogenic marker adiponectin also expressed PDGFRa (Fig. 5 a). Similarly, $83.40 \pm 3.67 \%$ of cells expressing PPAR- $y$ were positive for PDGFRa. There was also considerable accumulation of PDGFRa within perilipin+ fat cells (Fig. 4 d). Furthermore, oil red O staining showed strong overlap between mature adipocytes and PDGFRa-GFP+ cells (Fig. 6). These results suggest FAPs are the main source of adipocytes.

\section{Fibrogenic differentiation is found mainly in Tie2+ cells}

Similar methods of analysis were used to investigate fibrogenic differentiation. In our Tie2-GFP reporter mice, cells expressing Tie2-GFP overlapped strongly with vimentin (Fig. 7 a). Cells that were positive for vimentin showed $81.19 \pm 7.04 \%$ colocalization with Tie2 (Fig. 5 b). Staining for aSMA, a marker of myofibroblast formation, showed that $87.42 \pm 6.37 \%$ of aS$\mathrm{MA}^{+}$cells also expressed Tie2 (Fig. 7 b).

In the PDGFRa-GFP reporter mice, signal from endogenous PDGFRa-GFP was seen in only $25.28 \pm$ $3.44 \%$ of cells staining positive for vimentin (Fig. $8 a, b$ ). Quantification of fluorescence showed that $23.07 \pm$ $2.35 \%$ of the PDGFRa ${ }^{+}$cells colocalized with stains for aSMA (Fig. 7 b). Results from stains for both aS$\mathrm{MA}$ and vimentin suggest that cells expressing fibrotic markers mainly develop from a Tie2+ progenitor cell origin, although PDGFRa+ FAPs are also capable of differentiating into fibroblasts.

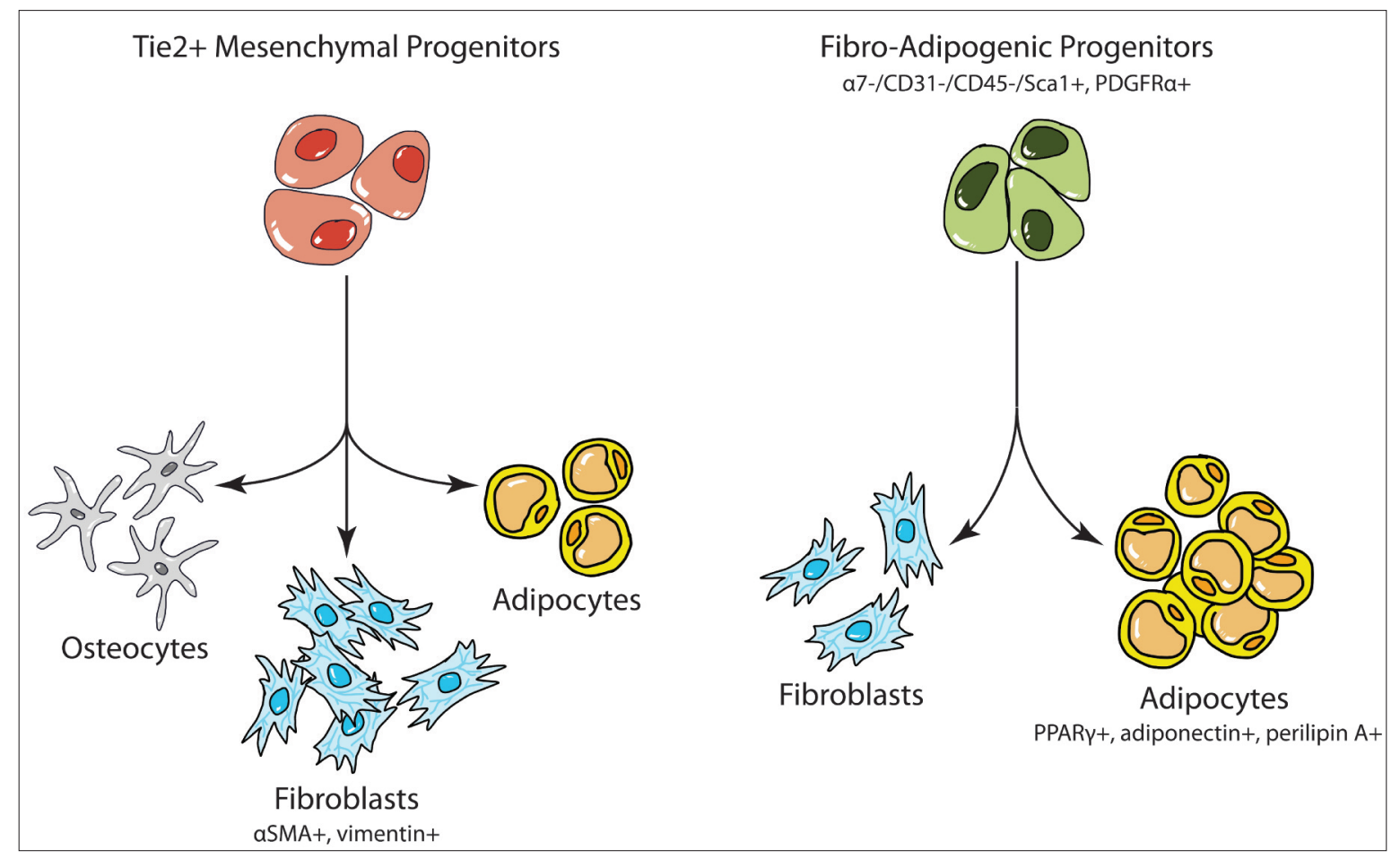

Figure 1. Proposed differentiation pathways for Tie2+ cells and FAP cells. 


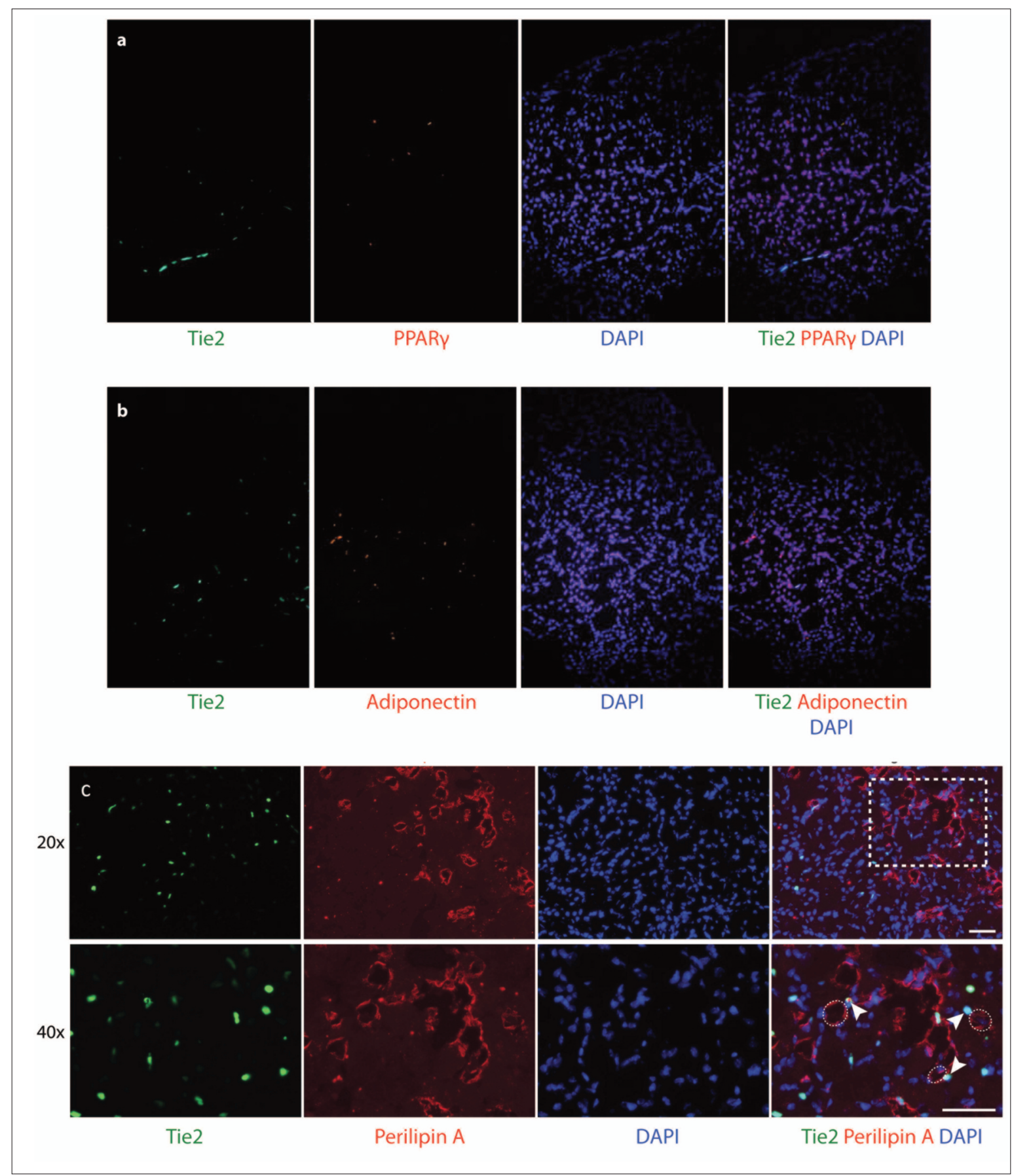

Figure 2. Behavior of Tie2+ cells in fatty infiltration of muscle six weeks post-injury. Tie2-GFP reporter mouse supraspinatus muscle sections were subjected to immunofluorescence staining for (a) middle-stage adipogenesis marker PPAR-g, (b) latestage adipogenesis marker adiponectin, and (c) perilipin A. Circles indicate area of lipid droplet as defined by perilipin A stain and arrowheads note adjacent Tie2+ stains. Dashed rectangle indicates area magnified at 40x; scale bar: $50 \mu \mathrm{m}$.

\section{Discussion}

The purpose of this study was to evaluate possible cellular sources of fibroblasts and adipocytes in RC muscle fibrosis and $\mathrm{FI}$ after tendon tears using re- porter mouse models. Rotator cuff $\mathrm{FI}$ is one of the most common muscle pathologies seen in orthopaedic patients, originally described and classified by Goutalier et al. ${ }^{3,4}$. FI has been a topic of significant interest in the orthopaedic community as it appears to strongly correlate with poor clinical out- 


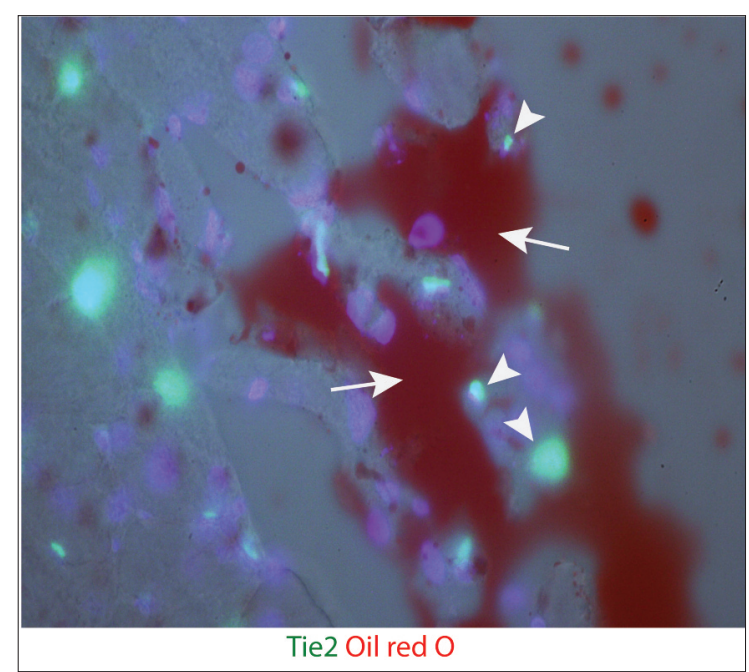

Figure 3. Mature adipocytes in Tie2-GFP reporter mouse supraspinatus muscle six weeks post-TT+DN operation stained with oil red $\mathrm{O}$. Arrows indicate fat cells. Arrowheads indicate Tie2+ ELCs.

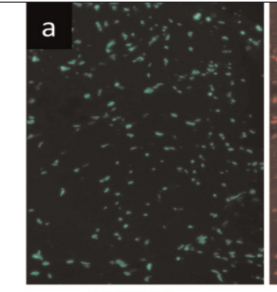

PDGFRa

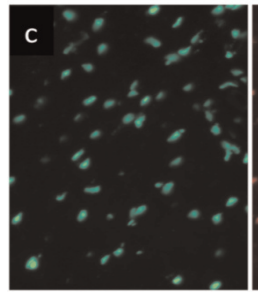

PDGFRa

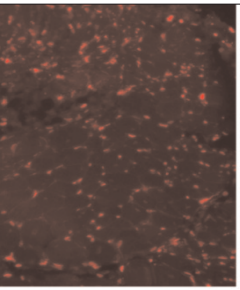

PPARY

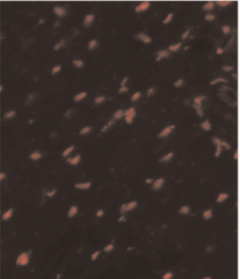

Adiponectin

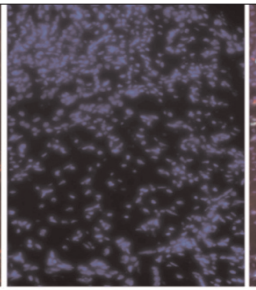

DAPI

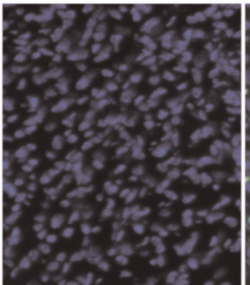

DAPI

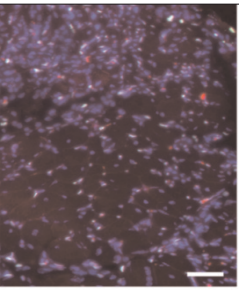

PDGFRa PPARY DAPI

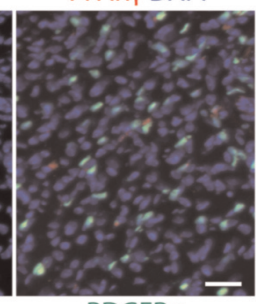

PDGFRa

Adiponectin DAPI
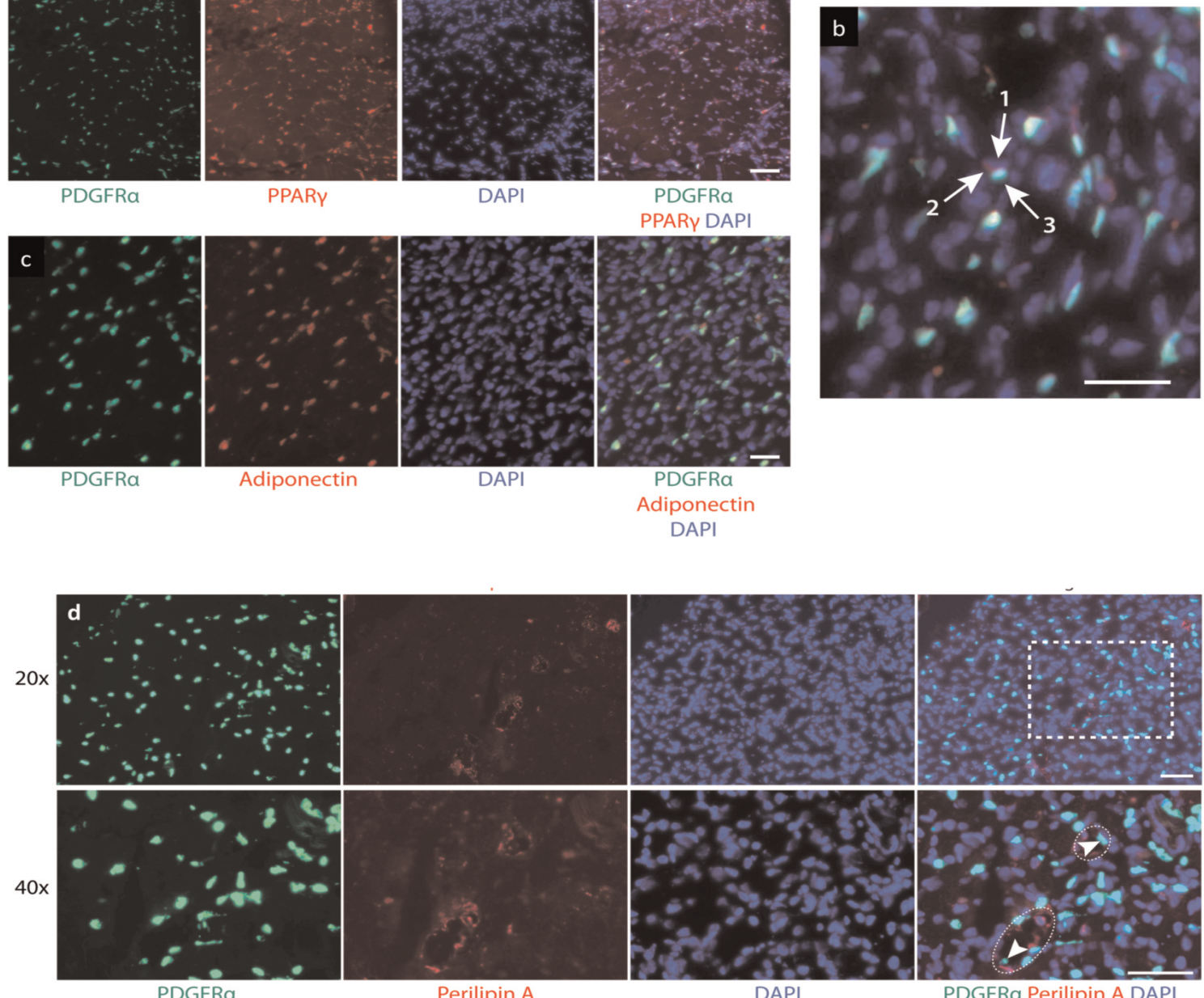

Perilipin A

DAPI

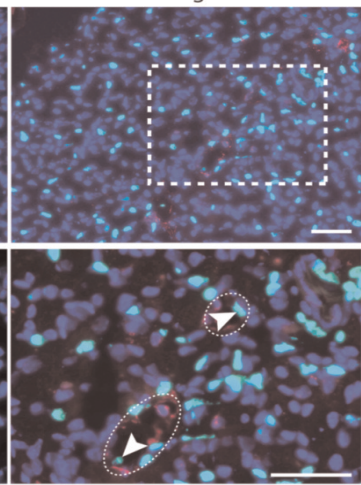

PDGFRa Perilipin A DAPI

Figure 4. Behavior of PDGFRa ${ }^{+}$cells in fatty infiltration of muscle six weeks post-injury. Muscle sections were subjected to immunofluorescence staining for middle-stage adipogenesis marker PPAR- $\gamma$ at (a) 20x and (b) 40x, scale bar: $25 \mu \mathrm{m}$; (c) late-stage adipogenesis marker adiponectin, scale bar: $50 \mu \mathrm{m}$; and (d) perilipin A, scale bar: $50 \mu \mathrm{m}$. Arrows in (b) indicate (1) nucleus, (2) PPAR- $\gamma$, and (3) PDGFRa. Circles in (d) highlight lipid droplets and arrowheads show PDGFRa signals within the fat cell. Dashed rectangle indicates area magnified at $40 x$. 


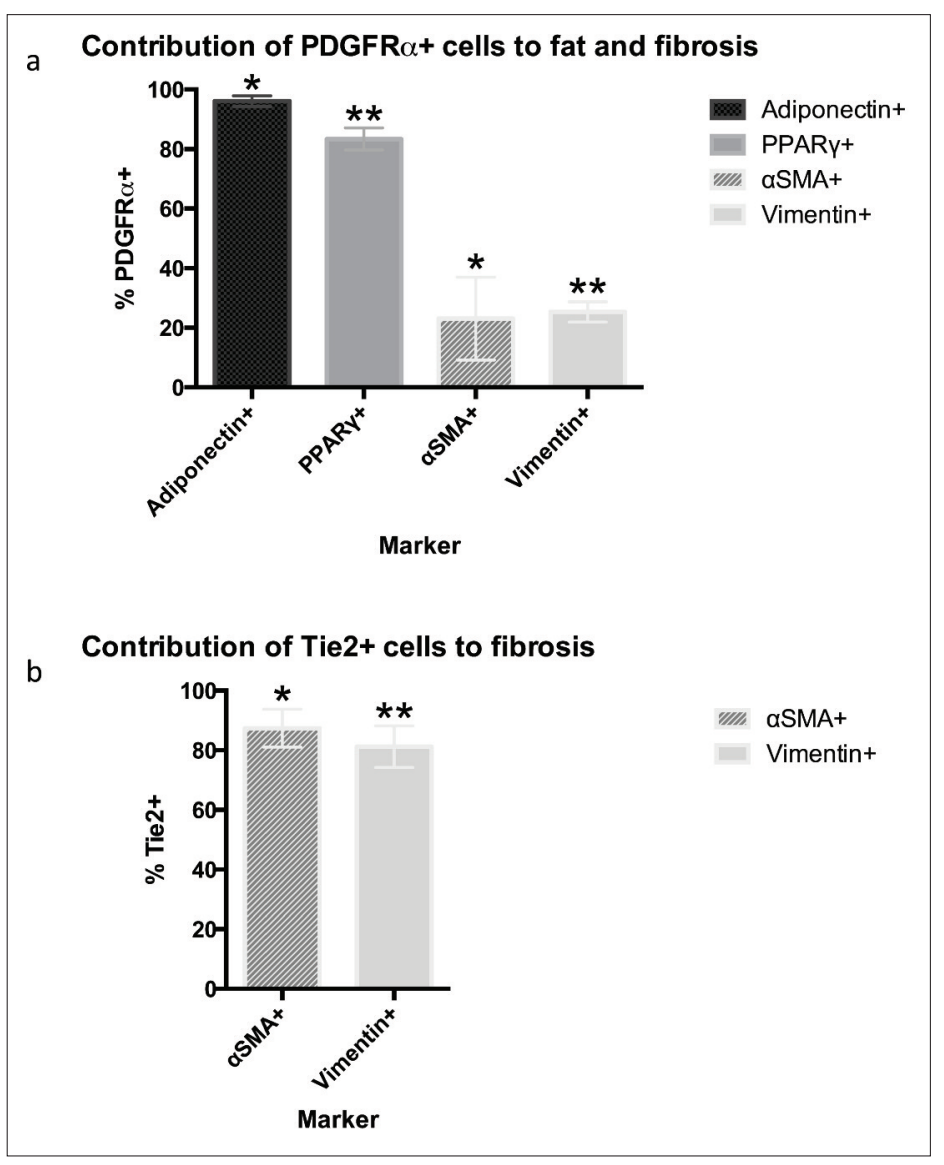

Figure 5. Contribution of Tie2+ and PDGFRa+ cells to formation of fatty infiltration and fibrosis. Data gathered as cell counts from two blinded independent reviewers across three samples $\left({ }^{*} p<0.05 ;{ }^{* *} p<0.001\right)$.

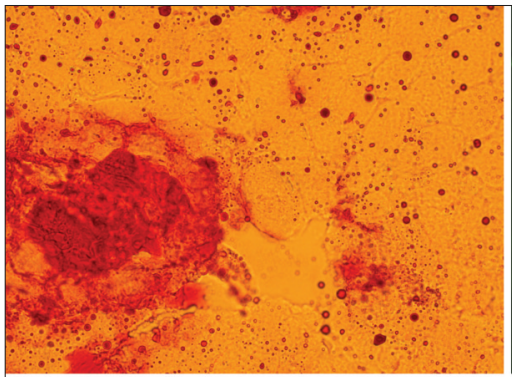

Oil red $\mathrm{O}$

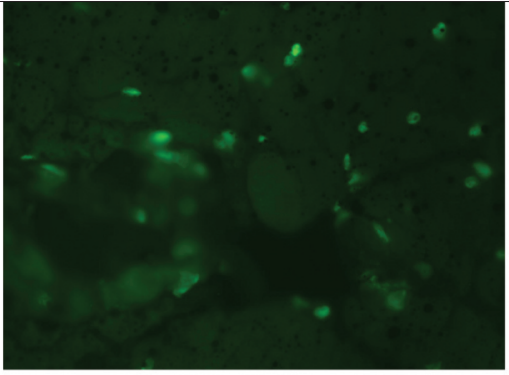

PDGFRa

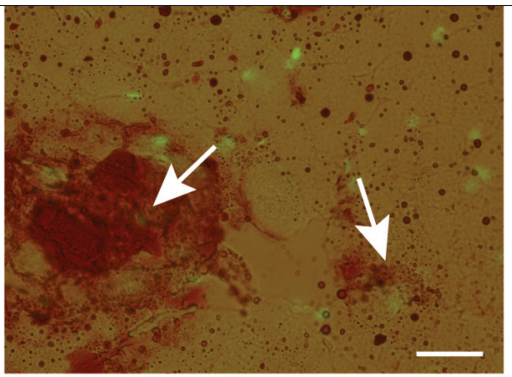

PDGFRa Oil red O

Figure 6. Colocalization of mature adipocytes and PDGFRa+ cells six weeks post-injury. Mouse supraspinatus muscle sections from PDGFRa-GFP reporter mice were subjected to oil red O staining. Arrows indicate overlap of the PDGFRa signal with fat cells. Scale bar: $100 \mu \mathrm{m}$.

comes and success rates of rotator cuff repairs. The degree of fatty infiltration is important not only for its effects on decreased muscle function, but also because there is a level of fatty infiltration that is irreversible even following repair. Patients graded with pathologic levels of fatty infiltration exhibit limited muscle strength of external rotation compared to those who maintain more rotator cuff musculature than fat. Thus, $\mathrm{FI}$ is an important predictor of disease progression and may potentially predict surgical outcome.
While much of muscle research has focused on intracellular changes that occur within the myocytes, the extracellular matrix (ECM) has a vital role in maintaining muscle homeostasis ${ }^{20}$. Injury to muscle often results in a dramatic increase in the amount of extracellular matrix production, resulting in muscle fibrosis. The ECM is a dynamic scaffold for the muscle and is made primarily of collagens. Type I and III collagen are the major forms, with type IV collagen surrounding the muscle cells in a basement membrane and adhering to a focal integrin adhesion complex. The 


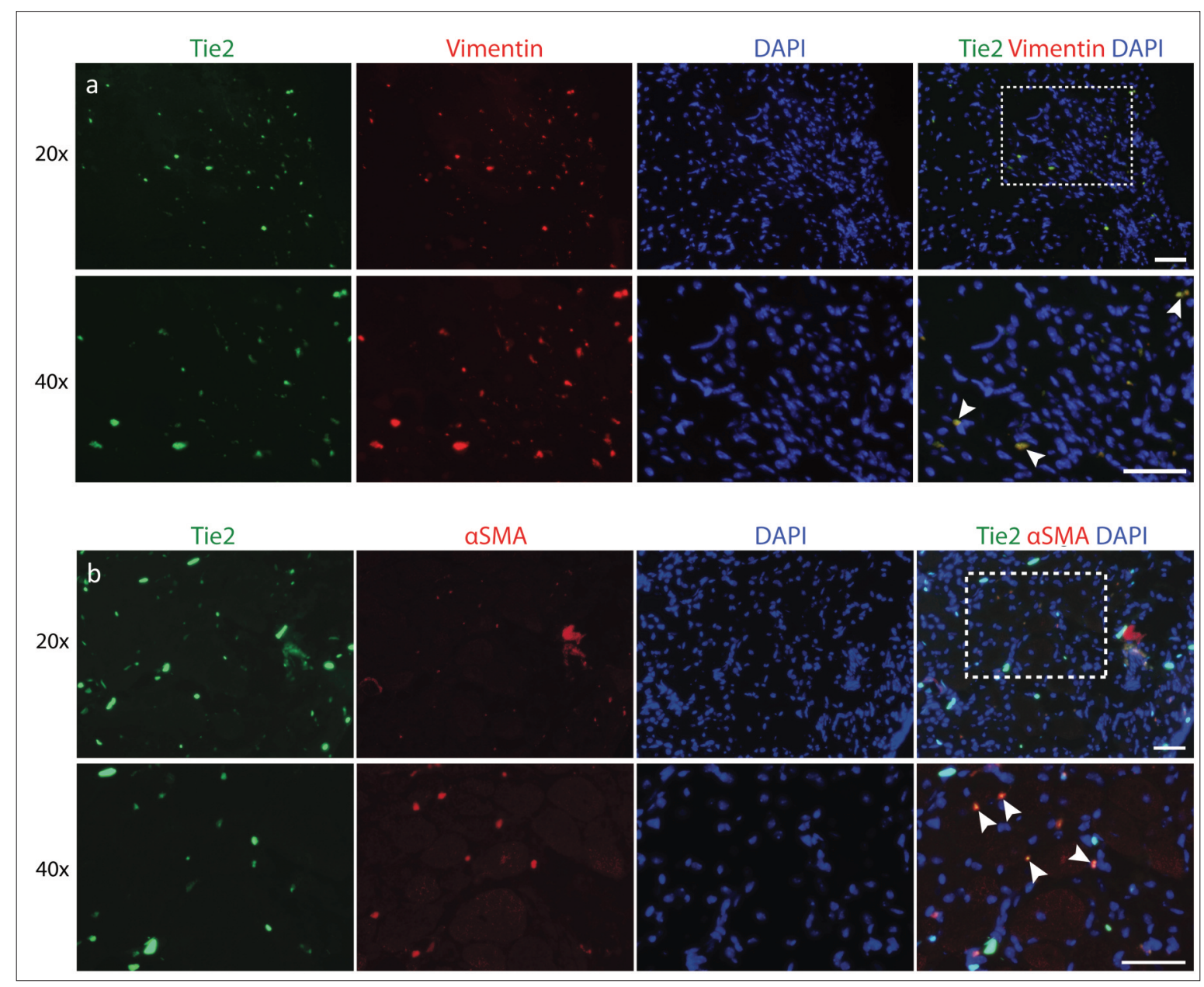

Figure 7. Colocalization of Tie2+ cells with fibrotic markers. Tie2-GFP reporter mouse muscle sections were analyzed for (a) vimentin and (b) aSMA. Arrowheads indicate regions of overlap. Scale bar: $50 \mu \mathrm{m}$.

complex association of the ECM to the intracellular components of muscle acts in most cases as an excellent transducer of mechanical activity, but is very sensitive to alterations in patterns of use. In most muscle injury models, including spaceflight, sarcopenia, denervation, and spinal cord injury, fibrosis is a central outcome of the injury process ${ }^{20}$ ENREF_14. In previous studies using a mouse model of massive $\mathrm{RC}$ tears, consistent fibrosis is seen ${ }^{18,21}$. It is postulated that increased fibrosis results in higher tension in the muscle in RC tears, thus making repair more challenging in chronic, retracted tears. In a clinical setting, fibrosis has been found in human $\mathrm{RC}$ tissue at time of repair and has been correlated with decreased force production and increased disruption of myofibril architecture 6, 22-24. Fibrosis has also been identified as a direct cause of muscle dysfunction in muscular dystrophy. Since fibrosis is not traditionally able to be imaged on advanced CT and MR scans, its influence on outcomes of $\mathrm{RC}$ repair may be underrepresented.

Despite the importance of these pathologies, little is known about the underlying etiology of both fibrosis and $\mathrm{FI}$ in rotator cuff muscles on a cellular and molecular mechanistic level. Previous studies have shown that the TGF-b pathway regulates fibrosis and that downstream components of the mTOR pathway mediate $\mathrm{FI}^{25}, 26$; however, the sources of these pathologies were not investigated. Our findings using Tie2GFP reporter mice indicated that $\mathrm{Tie}^{+}$muscle mesenchymal progenitor cells are the major source of fibroblasts in RC muscle fibrosis. Tie2 was previously considered to be a surface marker specific for endothelial cells, leading $\mathrm{Tie}^{+}$cells to be regarded as endothelial lineage cells (ELCs). However, a recent study demonstrated that Tie2 is also expressed in a separate population of muscle-resident progenitor cells $^{9}$. In that study, the Authors showed that $\mathrm{Tie}^{+}$ mesenchymal progenitors are the major source of osteocytes seen in muscle heterotopic ossification and have the potential to differentiate into multiple cell types, possibly including fibroblasts and adipocytes. Results from our study suggest that these Tie2+ progenitor cells do not form adipocytes but are the major cellular source of fibroblasts in rotator cuff muscle after injury. 


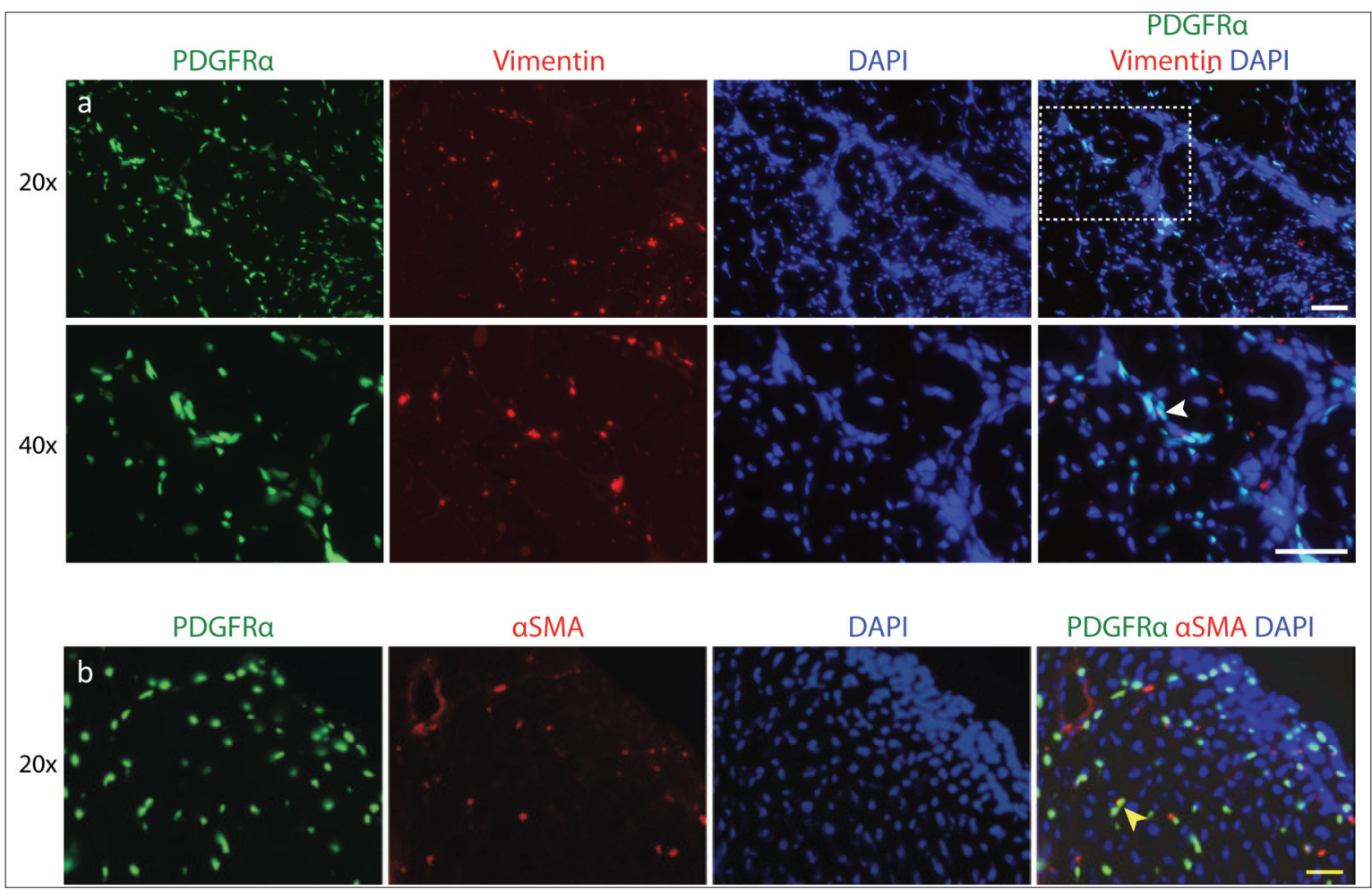

Figure 8. Colocalization of PDGFRa+ cells with fibrotic markers. Reporter mouse muscle sections were stained for (a) vimentin and (b) aSMA. Arrowheads show overlap with PDGFRa. Scale bar: $50 \mu \mathrm{m}$.

Furthermore, we identified PDGFRa+ FAPs as the major cellular source of adipocytes in rotator cuff muscle after tendon tears in our mouse model. The FAP cell population was first discovered by two separate groups in $2010^{11}, 12$. Using different cell surface markers, these two groups discovered a muscle-resident non-myogenic progenitor population that did not differentiate into myocytes in vivo or in vitro. Instead, these FAP cells were found to differentiate into fibroblasts and adipocytes under certain circumstances, such as chemical-induced muscle injury. One group further demonstrated that FAPs are characterized by the surface marker platelet-derived growth factor receptor alpha (PDGFRa) in both human and rodent skeletal muscles ${ }^{13},{ }^{14}$. Our immunofluorescence staining and histology suggest that FAPs are the major cellular source of adipocytes in rotator cuff muscle $\mathrm{FI}$.

Identification of the major cellular sources of rotator cuff muscle fibrosis and FI may help in the development of cellular targeted treatment for these diseases. FAP cells have recently been studied in a mouse model of muscular dystrophy, and results of this study provide insight into the plasticity of this cell line and the potential to drive this stem cell population to promote myogenesis in $\mathrm{RC}$ repair. A previous study found that HDAC inhibition induced core components of myogenic transcriptional machinery (MyoD and BAF60C) and upregulated multiple myogenic miRNAs to direct pro-myogenic differentiation and suppress the fibro-adipogenic phenotype ${ }^{27}$. Similar approaches may be considered in rotator cuff tear repair surgeries to promote $\mathrm{Tie}^{+}$cells and FAPs away from fibrogenic and adipogenic fates towards a more myogenic phenotype.

There are several potential limitations in this study. First, the PDGFRa-GFP reporter mice used in this study were not an inducible reporter mouse strain. An advantage of inducible reporter mice compared to non-inducible reporter mice is that the expression of the reporter protein (here, GFP) can be turned on and off at will. The PDGFRa-GFP reporter mouse strain used in this study has been used previously for in vivo cell tracing for adipogenesis ${ }^{16}, 17$. PDGFRa is not an adipocyte cell surface marker. Thus, it is unlikely that GFP-tagged PDGFRa is being produced by adipocytes from cellular origins other than FAPs. Second, due to the limited source of transgenic reporter mice, a relatively small sample size $(n=6)$ was used in this study. However, our results showed very similar staining patterns between samples. Thus, we believe our result is representative. Third, the Tie2GFP reporter mice do not distinguish between endothelial and non-endothelial $\mathrm{Tie}^{+}$progenitors in muscle. However, previous studies showed that nonendothelial $\mathrm{Tie}^{+}$cells, rather than endothelial $\mathrm{Tie}^{+}$ cells, possess multiple differentiation potentials. Though further work is needed, we believe Tie2+ muscle mesenchymal progenitor cells, rather than $\mathrm{Tie}^{+}$endothelial cells, are the major source of fibrob- 
lasts in RC muscles after tendon tears. Lastly, our mouse model involves transection of the suprascapular nerve. In humans, it is theorized that injury to the rotator cuff creates increased tension on the suprascapular nerve, which places the nerve at risk for injury ${ }^{28}$. This is a different mechanism of injury to the nerve than is seen clinically in rotator cuff tears; however, this mouse model has been shown to mimic pathophysiologic changes seen after a rotator cuff injury in previous studies ${ }^{18,29}$.

In summary, results from this study suggest that Tie $2^{+}$progenitor cells are the major source of fibroblasts and FAPs are the major cellular source of adipocytes in RC muscle following tendon tears. Future work will be needed to further examine the key pathways regulating fibrogenesis and adipogenesis of these progenitor cell populations in rotator cuff muscles, ultimately leading to the development of novel targeted treatments for secondary muscle degeneration after $\mathrm{RC}$ tears.

\section{Conclusion}

Rotator cuff tears are among the most common injuries seen by orthopedic surgeons and can result in secondary muscle degeneration that persists even after repair. The development of pathologies such as fatty infiltration and fibrosis can affect the outcome of repair; however, the cellular source of these complications has not previously been explored. Results from this study suggest that $\mathrm{Tie}^{+}$progenitor cells are the major source of fibroblasts and FAPs are the major cellular source of adipocytes contributing to degeneration in rotator cuff muscle following injury. This study takes the first step towards the development of targeted treatments to prevent secondary muscle degeneration after rotator cuff injury. Having identified these two populations, future work will be needed to further examine the key pathways regulating fibrogenesis and adipogenesis of these progenitor cells.

\section{Conflict of interests}

The Authors declare that they have no conflict of interests regarding the publication of this paper.

\section{References}

1. Shen PH, Lien SB, Shen HC, Lee CH, Wu SS, Lin LC. Longterm functional outcomes after repair of rotator cuff tears correlated with atrophy of the supraspinatus muscles on magnetic resonance images. J Shoulder Elbow Surg. 2008;17(1 Suppl):1S-7S

2. Gladstone JN, Bishop JY, Lo IK, Flatow EL. Fatty infiltration and atrophy of the rotator cuff do not improve after rotator cuff repair and correlate with poor functional outcome. Am J Sports Med. 2007;35(5):719-728.

3. Goutallier D, Postel JM, Bernageau J, Lavau L, Voisin MC. Fatty muscle degeneration in cuff ruptures. Pre- and postoper- ative evaluation by CT scan. Clin Orthop Relat Res. 1994 (304):78-83.

4. Goutallier D, Postel JM, Bernageau J, Lavau L, Voisin MC. Fatty infiltration of disrupted rotator cuff muscles. Rev Rhum Engl Ed. 1995;62(6):415-422.

5. Osti L, Buda M, Del Buono A. Fatty infiltration of the shoulder: diagnosis and reversibility. Muscles Ligaments Tendons Journal. 2014;3(4):351-354.

6. Davis ME, Korn MA, Gumucio JP, et al. Simvastatin reduces fibrosis and protects against muscle weakness after massive rotator cuff tear. J Shoulder Elbow Surg. 2015;24(2):280-287.

7. Oak NR, Gumucio JP, Flood MD, et al. Inhibition of 5-LOX, COX-1, and COX-2 increases tendon healing and reduces muscle fibrosis and lipid accumulation after rotator cuff repair. Am J Sports Med. 2014;42(12):2860-2868.

8. Kim HM, Dahiya N, Teefey SA, Keener JD, Galatz LM, Yamaguchi K. Relationship of tear size and location to fatty degeneration of the rotator cuff. J Bone Joint Surg Am. 2010;92(4): 829-839.

9. Wosczyna MN, Biswas AA, Cogswell CA, Goldhamer DJ. Multipotent progenitors resident in the skeletal muscle interstitium exhibit robust BMP-dependent osteogenic activity and mediate heterotopic ossification. J Bone Miner Res. 2012;27(5): 1004-1017.

10. Natarajan A, Lemos DR, Rossi FM. Fibro/adipogenic progenitors: a double-edged sword in skeletal muscle regeneration. Cell Cycle. 2010;9(11):2045-2046.

11. Joe AW, Yi L, Natarajan A, et al. Muscle injury activates resident fibro/adipogenic progenitors that facilitate myogenesis. Nat Cell Biol. 2010;12(2):153-163.

12. Uezumi A, Fukada S, Yamamoto N, Takeda S, Tsuchida K. Mesenchymal progenitors distinct from satellite cells contribute to ectopic fat cell formation in skeletal muscle. Nature cell biology. 2010;12(2):143-152.

13. Uezumi $A$, Ito $T$, Morikawa $D$, et al. Fibrosis and adipogenesis originate from a common mesenchymal progenitor in skeletal muscle. J Cell Sci. 2011;124(Pt 21):3654-3664.

14. Uezumi A, Fukada S, Yamamoto N, et al. Identification and characterization of PDGFRalpha+ mesenchymal progenitors in human skeletal muscle. Cell Death Dis. 2014;5:e1186.

15. Padulo J, Oliva F, Frizziero A, Maffulli N. Muscles, Ligaments and Tendons Journal. Basic principles and recommendations in clinical and field science research. MLTJ. 2013;3(4):250252.

16. Berry R, Rodeheffer MS. Characterization of the adipocyte cellular lineage in vivo. Nat Cell Biol. 2013;15(3):302-308.

17. Lee YH, Petkova AP, Granneman JG. Identification of an adipogenic niche for adipose tissue remodeling and restoration. Cell Metab. 2013;18(3):355-367.

18. Liu X, Laron D, Natsuhara K, Manzano G, Kim HT, Feeley BT. A mouse model of massive rotator cuff tears. J Bone Joint Surg Am. 2012;94(7):e41.

19. Hemmingsen $M$, Vedel $S$, Skafte-Pedersen $P$, et al. The role of paracrine and autocrine signaling in the early phase of adipogenic differentiation of adipose-derived stem cells. PLoS One. 2013;8(5):e63638.

20. Lieber RL, Ward SR. Cellular Mechanisms of Tissue Fibrosis. 4. Structural and functional consequences of skeletal muscle fibrosis. Am J Physiol Cell Physiol. 2013;305(3):C241-252.

21. Kan L, Lounev VY, Pignolo RJ, et al. Substance $P$ signaling mediates BMP-dependent heterotopic ossification. J Cell Biochem. 2011;112(10):2759-2772.

22. Choo A, McCarthy M, Pichika R, et al. Muscle gene expression patterns in human rotator cuff pathology. J Bone Joint Surg Am. 2014;96(18):1558-1565.

23. Sato EJ, Killian ML, Choi AJ, et al. Skeletal muscle fibrosis and stiffness increase after rotator cuff tendon injury and neuromuscular compromise in a rat model. J Orthop Res. 2014;32 
(9):1111-1116

24. Chillemi C, Petrozza V, Garro L, et al. Rotator cuff re-tear or non-healing: histopathological aspects and predictive factors. Knee Surg Sports Traumatol Arthrosc. 2011;19(9):1588-1596.

25. Joshi SK, Liu X, Samagh SP, et al. mTOR regulates fatty infiltration through SREBP-1 and PPARgamma after a combined massive rotator cuff tear and suprascapular nerve injury in rats. J Orthop Res. 2013;31(5):724-730.

26. Liu X, Joshi SK, Ravishankar B, Laron D, Kim HT, Feeley BT. Upregulation of transforming growth factor-beta signaling in a rat model of rotator cuff tears. J Shoulder Elbow Surg. 2014;23 (11):1709-1716.
27. Mozzetta C, Consalvi S, Saccone V, et al. Fibroadipogenic progenitors mediate the ability of HDAC inhibitors to promote regeneration in dystrophic muscles of young, but not old $\mathrm{Mdx}$ mice. EMBO Mol Med. 2013;5(4):626-639.

28. Albritton MJ, Graham RD, Richards RS 2nd, Basamania CJ. An anatomic study of the effects on the suprascapular nerve due to retraction of the supraspinatus muscle after a rotator cuff tear. Journal of Shoulder and Elbow Surgery. 2003;12: 497-500.

29. Liu X, Joshi S, Ravishankar B, Laron D, Kim HT, Feeley BT. Bone morphogenic protein signaling in rotator cuff muscle atrophy and fatty infiltration. Muscles, Ligaments and Tendons Journal. 2015;5(2):113-119. 